\title{
Carbon nanotubes as VEGF carriers to improve the early vascularization of porcine small intestinal submucosa in abdominal wall defect repair
}

This article was published in the following Dove Press journal:

International Journal of Nanomedicine

10 March 2014

Number of times this article has been viewed

\author{
Zhengni Liu',* \\ Xueyi Feng',* \\ Huichun Wang' \\ Jun Ma' \\ Wei Liu ${ }^{3}$ \\ Daxiang Cui ${ }^{4}$ \\ Yan Gu' \\ Rui Tang'
}

'Department of General Surgery, Shanghai Ninth People's Hospital, Hernia and Abdominal Wall Disease Center, Shanghai Jiao Tong University School of Medicine, Shanghai, People's Republic of China; ${ }^{2}$ Department of General Surgery, Lu'an People's Hospital, Lu'an Affiliated Hospital of Anhui Medical University, Lu'an, Province Anhui, People's Republic of China; ${ }^{3}$ Department of Plastic and Reconstructive Surgery, Shanghai Ninth People's Hospital, Shanghai Jiao Tong University School of Medicine, Shanghai Key Laboratory of Tissue Engineering, Shanghai, People's Republic of China; ${ }^{4}$ Institute of Nano Biomedicine and Engineering, Key Laboratory for Thin Film and Microfabrication Technology of the Ministry of Education, Research Institute of Micro/Nano Science and Technology, Bio-X Center, Shanghai Jiao Tong University, Shanghai, People's Republic of China

*These authors contributed equally to this work

Correspondence: Rui Tang Department of General Surgery, Shanghai Ninth People's Hospital, Hernia and Abdominal Wall Disease Center, Shanghai Jiao Tong University School of Medicine, Shanghai 2000II, People's Republic of China

Tel +862 2I 2327 I699 ext 5146

Fax +862163087768

Email kevintown@126.com
Abstract: Insufficient early vascularization in biological meshes, resulting in limited host tissue incorporation, is thought to be the primary cause for the failure of abdominal wall defect repair after implantation. The sustained release of exogenous angiogenic factors from a biocompatible nanomaterial might be a way to overcome this limitation. In the study reported here, multiwalled carbon nanotubes (MWNT) were functionalized by plasma polymerization to deliver vascular endothelial growth factor ${ }_{165}\left(\mathrm{VEGF}_{165}\right)$. The novel VEGF ${ }_{165}$-controlled released system was incorporated into porcine small intestinal submucosa (PSIS) to construct a composite

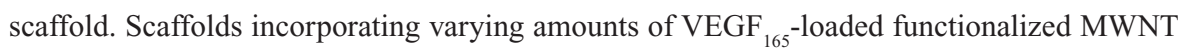
were characterized in vitro. At 5 weight percent MWNT, the scaffolds exhibited optimal properties and were implanted in rats to repair abdominal wall defects. PSIS scaffolds incorporating VEGF $_{165}$-loaded MWNT (VEGF-MWNT-PSIS) contributed to early vascularization from 2-12 weeks postimplantation and obtained more effective collagen deposition and exhibited improved tensile strength at 24 weeks postimplantation compared to PSIS or PSIS scaffolds, incorporating MWNT without VEGF $_{165}$ loading (MWNT-PSIS).

Keywords: vascular endothelial growth factor ${ }_{165}$, controlled release, multi-walled carbon nanotube, early vascularization

\section{Introduction}

Synthetic or biological materials have been used to repair the abdominal wall defects caused by the extensive surgical tumor resections or the loss of large amounts of tissue following traumatic injury. ${ }^{1}$ The use of synthetic materials, including polypropylene, to repair such defects gives rise to the deposition of nonspecific fibrotic tissue, thereby leading to complications, such as bowel obstruction. ${ }^{2-4}$ On the other hand, biological materials lead to the regeneration of new site-specific tissue that is similar to the original host tissue in terms of histology and function. ${ }^{5}$ This is known as tissue endogenous regeneration. Therefore, biological materials are considered ideal implants for repairing abdominal wall defects.

As a widely used biological material, porcine small intestinal submucosa (PSIS) is prepared from the original material by removing all cells and retaining the threedimensional extracellular matrix. The extracellular matrix acts as a scaffold onto which cells migrate and proliferate after an attachment to the substratum. The tensile strength of a hernia repair using PSIS is achieved via host tissue incorporation. ${ }^{6,7}$ However, the lack of early vascularization limits host tissue incorporation following implantation, weakening the mechanical strength of the defect site, ${ }^{8}$ and leading to implant failure. ${ }^{9,10}$ 
Delivery of exogenous angiogenic factors - such as VEGF - might facilitate early vascularization and promote efficient host integration.

VEGF is a potent angiogenic factor that is rapidly degraded due to its short half-life. ${ }^{11}$ Therefore, VEGF encapsulated in microspheres or nanospheres that undergo gel-sol transition are used for its delivery. However, encapsulated VEGF is often uncontrollably released from the carrier matrix. ${ }^{12,13}$ Using fibrin matrices, Wilcke et al ${ }^{14}$ developed a delayed-release system for the delivery of $\mathrm{VEGF}_{165}$ and basic fibroblast growth factor to repair full-skin defects on the backs of mice. After the first 48 hours, the concentration of $\mathrm{VEGF}_{165}$ released decreased by $50 \%$ compared to that in the first 24 hours. Such rapid releasing might be prevented by the binding of the growth factors to biodegradable polymeric matrices. Then, the sustained releasing of the growth factors will be obtained under the control of locally biodegradable polymeric matrices. ${ }^{15}$ However, owing to its biodegradable and polyporous characteristics, achieving the controlled release of VEGF from PSIS remains a challenge. Abdominal wall defect repair requires the sustained release of VEGF at high concentrations to ensure early vascularization.

The development of nanomaterials has allowed the controlled delivery of therapeutic peptides. Using multiwalled carbon nanotubes (MWNT), we designed a system for the controlled release of therapeutic molecules to achieve early vascularization. We chose MWNT because of its hollow and cylindrical structure that could readily carry and deliver drug molecules (for example, amphotericin B or methotrexate). ${ }^{16}$ Besides that, plasma polymerization is an effective method for nanotube functionalization on the surface of substrates with a polymer coating. ${ }^{17}$ In this study, MWNT were prepared through plasma polymerization to carry and release $\mathrm{VEGF}_{165}$ which makes it possible to deposit the uniform poly(lactic-co-glycolic) acid (PLGA) coating on MWNT firmly and steadily. Furthermore, owing to the hollow and cylindrical structure with the wide contact surface of MWNT, VEGF can be not only loaded into the tube, but it can be adhered on the sidewall of MWNT with noncovalent integration. Hence, the structure and the coating-relevant plasma polymerization of VEGF-MWNT that we developed makes the system totally different from the PLGA-VEGF microsphere.

We hypothesized that $\mathrm{VEGF}_{165}$ could be released from the hollow tube structures of MWNT during the slow degradation of the PLGA used to coat the material, thereby avoiding the uncontrolled burst release of growth factor from the carrier matrix, such as PLGA-VEGF microsphere, and supporting prolonged and sustained release. Such a system could then support early neovascularization, leading to better tissue regeneration.
Consequently, we constructed a composite scaffold with an optimal content of $\mathrm{VEGF}_{165}-\mathrm{MWNT}$ that was capable of carrying and releasing $\mathrm{VEGF}_{165}$ without inhibiting cell proliferation (Figure 1A). Such composite scaffolds were implanted into the abdominal wall defect sites in rats to evaluate their efficacy in inducing early neovascularization.

\section{Methods}

All experiments were conducted according to local guidelines on the ethical use of animals and the National Institutes of Health's Guide for the Care and Use of Laboratory Animals (US National Institutes of Health publication number 85-23, revised 1996). Animal study protocols were approved by an institutional review committee at the Shanghai Jiao Tong University School of Medicine, Shanghai, People's Republic of China.

\section{MWNT functionalization}

The versatile features of MWNT are functionalized, such as the multipurpose innovative carriers for drug delivery and diagnostic applications via physicochemical treatment. In our study, it means the MWNT are purified and treated by plasma polymerization to obtain the ability to carry and release $\mathrm{VEGF}_{165}$. Raw MWNT (Wako Pure Chemical Industries, Ltd, Osaka, Japan) was purchased and purified as previously reported. ${ }^{18} \mathrm{VEGF}_{165}$ was purchased from R\&D Systems, Inc. (Minneapolis, MN, USA). Recombinant human VEGF $_{165}$ was loaded into purified MWNT at a ratio of $1: 10,000(0.1 \mu \mathrm{g}: 1 \mathrm{mg})$ by solution sonication for 30 minutes. The suspensions were then freeze-dried for 48 hours. The VEGF 165 -loaded MWNT were coated with PLGA film by plasma polymerization to prepare the $\mathrm{VEGF}_{165}$-releasing device, as reported previously. ${ }^{19}$ The ratio of lactic acid to glycolic acid was 1:2. After plasma polymerization for 12 hours, the novel MWNT were characterized using high-resolution transmission electron microscopy (HRTEM). The amount of VEGF $_{165}$ loaded into MWNT was analyzed by human VEGF enzyme-linked immunosorbent assay (ELISA) kits (Quantikine ${ }^{\circledR}$ Colorimetric Sandwich ELISAs, R\&D Systems), according to the manufacturer's instructions.

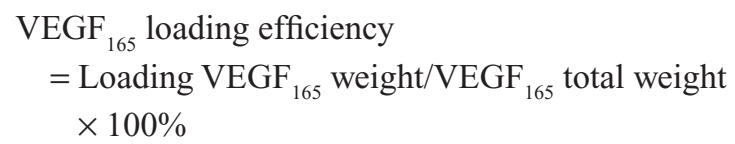

\section{Scaffold preparation}

The PSIS scaffolds were prepared as previously described. ${ }^{20}$ In brief, fresh porcine small intestine was obtained from 
A
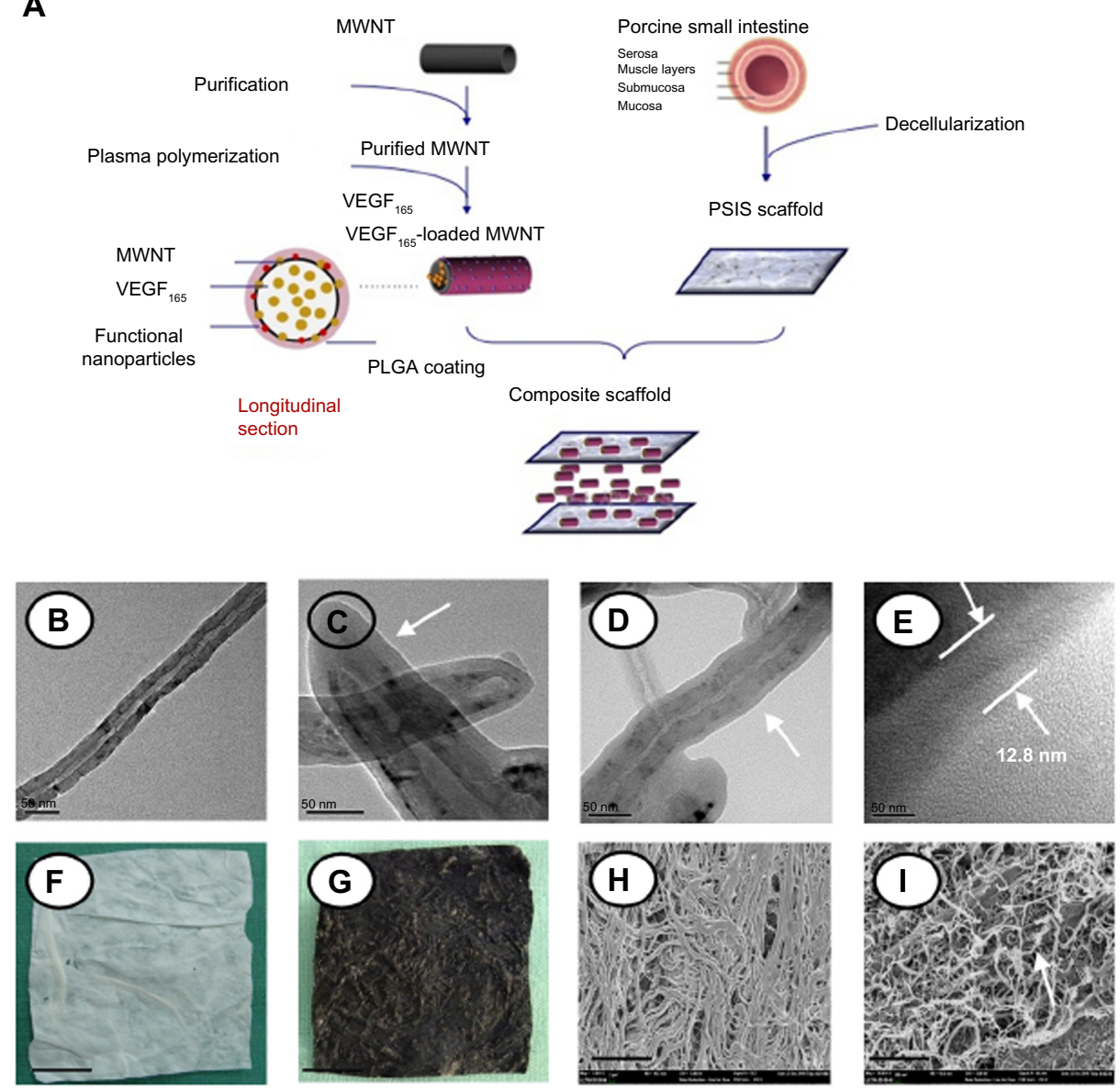

Figure I Examination of composite scaffold.

Notes: (A) Preparation of composite scaffold. (B) TEM images of MWNT showed a single straight (not bowed) of purified MWNT. HRTEM images showed that a thin film of plasma-deposited PLGA polymer was coated both on the tip (C) and on the side wall of the MWNT (D). An ultrathin PLGA film of approximately I2.8 nm was seen on the outer surface of the nanotube after plasma polymerization (E). PSIS scaffold and composite scaffold containing $5 \%$ MWNT were prepared (F-G). The PSIS scaffold showed a loosely arranged meshwork $(\times 500)(\mathbf{H})$; whereas, the surface of the composite scaffold was coated with permeated MWNT (bright bands) on SEM examination $(\times 10,000)(\mathbf{I})$. The bars indicate: $100 \mathrm{~nm}$ in (B); $50 \mathrm{~nm}$ in (C and $\mathbf{D}) ; 10 \mathrm{~nm}$ in $(\mathbf{E}) ; \mathbf{I} \mathrm{cm}$ in (F and $\mathbf{G}) ; 20 \mu \mathrm{m}$ in $(\mathbf{H})$; and $2 \mu \mathrm{m}$ in (I).

Abbreviations: MWNT, multiwalled carbon nanotubes; VEGF, vascular endothelial growth factor; PLGA, poly(lactic-co-glycolic) acid; PSIS, porcine small intestinal submucosa; TEM, transmission electron microscopy; HRTEM, high-resolution transmission electron microscopy; SEM, scanning electron micrography.

Fuxin abattoir (Shanghai, People's Republic of China). After flushing the intestinal contents, the serosal and muscular layers of the porcine small intestine were removed mechanically. Then, the decellularized treatment was done by shaking the small intestinal segments at $4^{\circ} \mathrm{C}$ in $1 \mathrm{~L}$ of $0.2 \%$ Triton $\mathrm{X}-100$ (Sigma-Aldrich, St Louis, MO, USA) containing $26.5 \mathrm{mmol} / \mathrm{L}$ ammonium hydroxide for 7 days. The two-layer PSIS grafts were trimmed into a size of $40 \times 30 \times 0.2 \mathrm{~mm}$ membranes.

Afterward, the $\mathrm{VEGF}_{165}$-releasing devices were suspended in $2 \mathrm{~mL}$ distilled water by ultrasonication to prepare the suspensions. Varying amounts of the suspension were integrated with the two-layer PSIS scaffold by dipping and sonication. Then, the $\mathrm{VEGF}_{165}-\mathrm{MWNT}$ contained composite scaffolds were freeze-dried. The structures of prepared PSIS scaffolds and composite scaffolds including
VEGF $_{165}-$ MWNT were observed by scanning electron micrography (SEM Ultra55, Carl Zeiss AG, Oberkochen, Germany). Scaffolds were then trimmed to a size of $40 \times 30 \times 0.2 \mathrm{~mm}$ and stored at $-80^{\circ} \mathrm{C}$.

\section{Evaluation of in vitro properties of composite scaffolds}

Group $\mathrm{A}$, group $\mathrm{B}$, group $\mathrm{C}$, and group $\mathrm{D}$, containing $1 \%, 3 \%$, $5 \%$, and $10 \%$ weight (mg per mg) of $\mathrm{VEGF}_{165}-\mathrm{MWNT}$ in the composite scaffolds, were assigned as four experimental groups. The PSIS scaffold without VEGF ${ }_{165}-\mathrm{MWNT}$ was assigned as the control group. The optimal content of VEGF $_{165}-$ MWNT was determined by analyzing VEGF release, bioactive property, biocompatibility, and the mechanical property of the composite scaffold. 


\section{Examination of controlled release} and bioactive property of VEGF ${ }_{165}$

To assess the amount of $\mathrm{VEGF}_{165}$ released, we incubated 50 $\mathrm{mg}$ of composite scaffolds from each group in $50 \mathrm{~mL}$ phosphate buffered saline at $37^{\circ} \mathrm{C}$ with occasional shaking $(120$ revolutions per minute). Then, $100 \mu \mathrm{L}$ aliquots of the incubation buffers were taken at 10 time points: 1 hour; 6 hours; 12 hours; and at 1 day; 2 days; 3 days; 4 days; 5 days; 6 days; and 7 days. The samples were analyzed by human VEGFELISA kits. The cumulative amounts of released $\mathrm{VEGF}_{165}$ were plotted against time to exhibit the in vitro $\mathrm{VEGF}_{165^{-}}$ releasing concentration.

The bioactivity of released VEGF $_{165}$ was assessed by endothelial cell proliferation assay. In this test, the PSIS and composite scaffolds were cultured with $10 \mathrm{~mL}$ of medium M 199 containing 10\% fetal bovine serum for 3 days to release the loaded $\mathrm{VEGF}_{165}$ sufficiently, and the leaching solutions were collected. In the next step, $100 \mu \mathrm{L}$ of human umbilical vein endothelial cell (HUVECs) (American Type Culture Collection [ATCC], Manassas, VA, USA) suspension in HUVEC growth medium (PromoCell GmbH, Heidelberg, Germany) was seeded onto individual wells of 96 -well plates at a density of $2 \times 10^{3}$ cells per $\mathrm{mL}$. Cultures were maintained under standard conditions for adherence. Following this, the culture medium was replaced with $100 \mu \mathrm{L}$ leaching solution. HUVECs cultured with PSIS scaffold leaching solution were assigned as the control group, while the HUVECs cultured with composite scaffolds leaching solution were assigned as the experimental groups. HUVECs were cultured serially and counted at 1 day, 3 days, 5 days, and 7 days without changing medium, using Cell Counting Kit-8 (CCK-8; Dojindo Molecular Technologies, Inc., Kumamoto, Japan), following manufacturer's instructions.

\section{Examination of biocompatibility}

Biocompatibility of the composite scaffold was determined by the fibroblasts' proliferation test. The fibroblasts were cocultured with PSIS scaffold as the negative control. Fibroblasts cultured in MWNT suspensions $(1 \mathrm{mg} / \mathrm{mL}$ ) were used as positive control. The fibroblast cell counting test was similar to that described for HUVECs.

\section{Examination of mechanical properties}

Mechanical properties of the $50 \times 10 \mathrm{~mm}$ samples were examined at room temperature using a uniaxial materials testing machine (Model 5542; Instron, Norwood, MA, USA), as described previously. ${ }^{20}$ The abdominal wall strengths in rats were also recorded.

\section{Evaluation of in vivo properties of composite scaffold \\ Experimental animals}

In addition, 60 male Sprague Dawley rats (weight, 200-250 g) (SLAC National Rodent Laboratory Animal Resources, Shanghai, People's Republic of China) were divided into three groups, according to the nature of the scaffold implanted. The PSIS and MWNT-PSIS groups were assigned as negative control groups, and the $\mathrm{VEGF}_{165}-\mathrm{MWNT}$-PSIS group was assigned as the experimental group. Each group had 20 rats. The rats were housed in accordance with current national guidelines on animal welfare. The environment of cages was maintained at $18^{\circ} \mathrm{C}-26^{\circ} \mathrm{C}$, with a relative humidity of $30 \%-70 \%$.

\section{Surgical procedure}

Rats were anesthetized by intramuscular injection of ketamine at the dose of $60 \mathrm{mg} / \mathrm{kg}$. The abdominal skin was shaved and disinfected with a povidone-iodine solution. A longitudinal midline abdominal incision of $50 \mathrm{~mm}$ length was made, and a complete defect - including fascia, muscles, and peritoneum - was created (with a $30 \times 20 \mathrm{~mm}$ size). Subsequently, a sterile scaffold piece with dimensions of $40 \times 30 \mathrm{~mm}$ was implanted into each rat to repair the defect in a sublay manner. Each layer of the defects was stitched up and overlapped with the edge of the scaffolds for $5 \mathrm{~mm}$ on each side. The scaffold was fixed with 3-0 polypropylene with interrupted sutures. Skin was closed with continuous sutures with 2-0 absorbable polydioxanone (Ethicon, Inc., Somerville, NJ, USA). Antibiotics were not administered during or after the implantation.

\section{Macroscopic observations}

Daily examination included study of the animals' eating, drinking, urination, and bowel movements, palpation of the implant sites for integrity, and visual inspection of the abdominal body wall for asymmetry or abnormal contours. Four rats from each group were randomly sacrificed in sequence at 1 week, 2 weeks, 4 weeks, 12 weeks, and 24 weeks postimplantation. Rats were suspended vertically and horizontally to assess the status of the repaired abdominal wall. The implants on the subcutaneous and visceral sides were examined for the presence of seromas, hematomas, and infection. The site dimensions were measured to assess possible intracorporeal shrinkage or expansion. An established grading scale, as described by Jenkins et al, ${ }^{21}$ was used to semiquantitatively grade adhesion tenacity (Table 1). The thickness of the implant was estimated by ten random 
Table I Semiquantitative adhesion scoring system

\begin{tabular}{lll}
\hline Score & Tenacity & Surface area \\
\hline 0 & No adhesions & No adhesions \\
I & Minimal adhesions freed by blunt dissection & $<25 \%$ \\
2 & Moderate adhesions freed by aggressive & $<50 \%$ \\
& dissection & \\
3 & Dense adhesions freed by sharp dissection & $<75 \%$ \\
4 & - & $\geq 75 \%$ \\
\hline
\end{tabular}

Note: Data from Jenkins et al. ${ }^{21}$

measurements over the central part of the implant 24 weeks after implantation.

\section{Histological observations}

The implants and a $1 \mathrm{~cm}$ strip of surrounding host tissue were resected. Sections of the explant were fixed with $10 \%$ formalin, paraffin embedded, and sectioned into $5 \mu \mathrm{m}$ slices. Immunostaining was performed to examine neovascularization of the implants at various stages of regeneration. Samples were incubated with anti-von Willebrand factor antibody (vWF; 1:1,000; Abcam, Cambridge, MA, USA) to evaluate the density of capillary vessels. Sections on which immunohistochemistry was performed without primary antibody served as negative controls. The average percentage of areas with blood vessels $\left(\% \mathrm{~A}_{\mathrm{bv}}\right)$ was calculated for ten randomly selected fields $(\times 400)$, using Image-Pro Plus software 6.0 (Media Cybernetics, Inc., Silver Spring, MD, USA), according to the following equation:

Percentage of areas with blood vessels

$=$ Capillary vessels area/total tissue area.

Qualitative assessment of collagen deposition was performed by using Masson's trichrome stain. Hematoxylin and eosin staining was performed to quantify the presence of foreign body giant cells, polymorphonuclear cells and mononuclear cells. The scale used was similar to that described by Badylak et al (Table 2). ${ }^{22}$ Five fields per slice were counted

Table 2 Histologic scoring criteria for microscopic examination

\begin{tabular}{|c|c|c|c|c|}
\hline \multirow[t]{2}{*}{ Category } & \multicolumn{4}{|l|}{ Score } \\
\hline & 0 & I & 2 & 3 \\
\hline $\begin{array}{l}\text { Foreign body } \\
\text { giant cells }\end{array}$ & 0 & $\mathrm{I}-5$ & $6-10$ & $>10$ \\
\hline $\begin{array}{l}\text { Polymorphonuclear } \\
\text { cells }\end{array}$ & 0 & $\mathrm{I}-5$ & $6-10$ & $>10$ \\
\hline Mononuclear cells & 0 & $\mathrm{I}-5$ & $6-10$ & $>10$ \\
\hline \multicolumn{5}{|l|}{ Collagen } \\
\hline Organization & $\begin{array}{l}\text { Totally } \\
\text { disorganized }\end{array}$ & $\begin{array}{l}\text { Slightly } \\
\text { organized }\end{array}$ & $\begin{array}{l}\text { Moderately } \\
\text { organized }\end{array}$ & $\begin{array}{l}\text { Well- } \\
\text { organized }\end{array}$ \\
\hline Amount & 0 & Mild & Moderate & Abundant \\
\hline
\end{tabular}

Note: Data from Badylak et al. ${ }^{22}$ under 400× magnification (E600; Nikon Corporation, Tokyo, Japan). One pathologist, who was blinded to the tested materials, performed all evaluations.

\section{Examination of mechanical properties}

The load of $1 \mathrm{~cm}$ wide explants was measured by mechanical evaluation as described. The tensile strength of the explant was calculated from the stress-strain curves.

\section{Statistical analysis}

Kolmogorov-Smirnov tests were performed for ranked data. Measurement data were recorded as mean \pm standard deviation. The differences in quantitative parameters between experimental and control groups were analyzed by one-way analysis of variance followed by the Bonferroni post hoc test. SPSS version 13.0 software (SPSS Inc., Chicago, IL, USA) was used for statistical analysis. $P<0.05$ was considered as statistically significant.

\section{Results \\ MWNT functionalization}

The transmission electron microscopy examination showed that acid treatment improved the reunion and winding phenomenon in raw MWNT (Figure 1B). After VEGF ${ }_{165}$ loading, the surface functionalization of MWNT was achieved by depositing PLGA film onto the nanotubes by plasma polymerization. The microstructure of the composites was characterized using HRTEM (Figure $1 \mathrm{C}-\mathrm{E}$ ). The results of ELISA showed that the $\mathrm{VEGF}_{165}$ loading efficiency was $15.1 \% \pm 0.6 \%$.

\section{Scaffold preparation}

The PSIS scaffold and the composite scaffolds were successfully prepared and their structures were examined by scanning electron micrography (Figure $1 \mathrm{~F}-\mathrm{I}$ ).

\section{Evaluation of properties of composite scaffold in vitro}

The composite scaffolds were divided into four experimental groups, according to the $\mathrm{VEGF}_{165}-\mathrm{MWNT}$ content, to assess the $\mathrm{VEGF}_{165}$ releasing and bioactive properties, mechanical properties, and biocompatibility in vitro.

\section{Examination of released amount and bioactive property of VEGF ${ }_{165}$} The cumulative release of $\mathrm{VEGF}_{165}$ in four experimental and one control groups is shown in Figure 2A. In groups A and B, the cumulative concentration of $\mathrm{VEGF}_{165}$ released after 7 days 
A
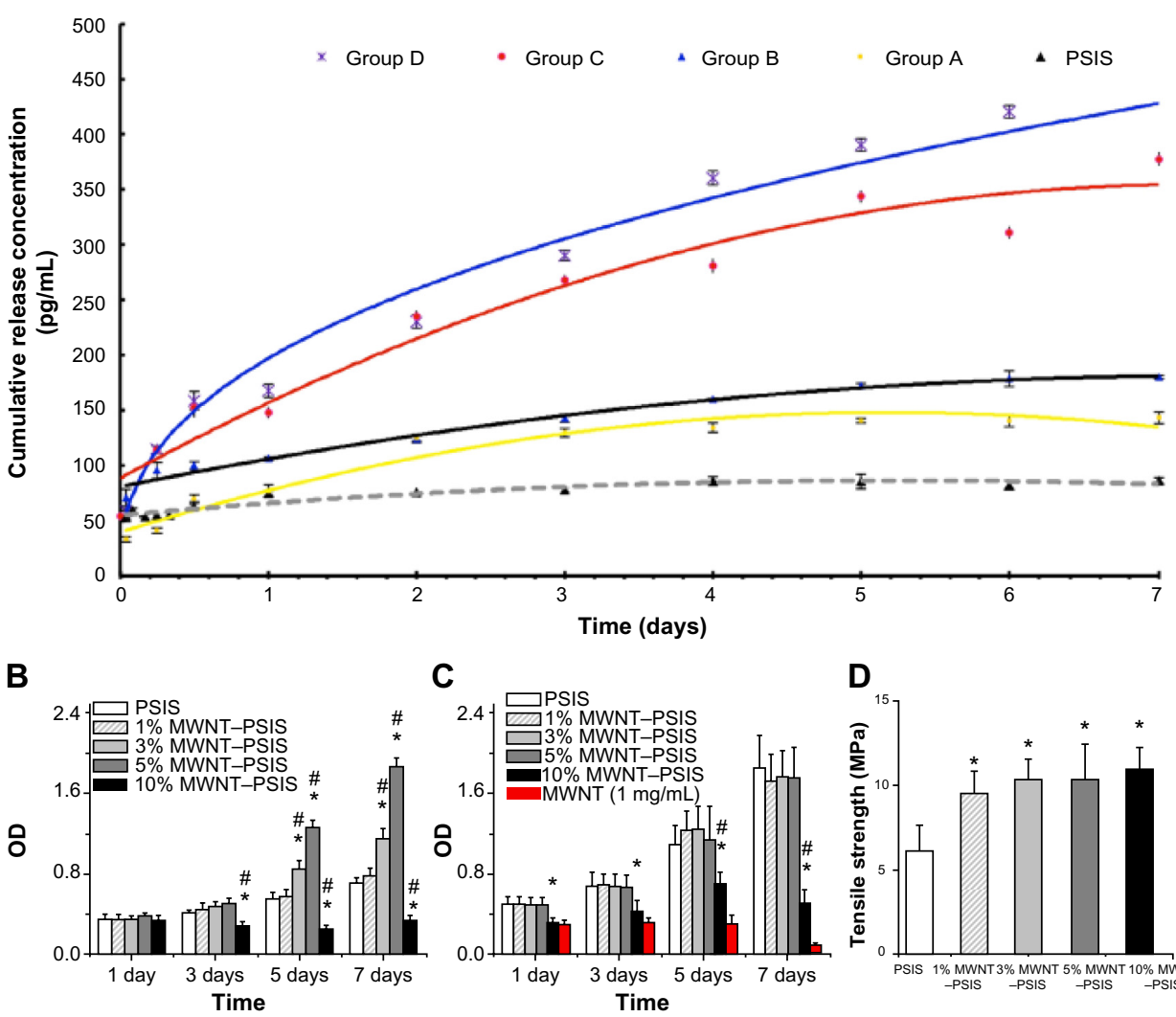

C

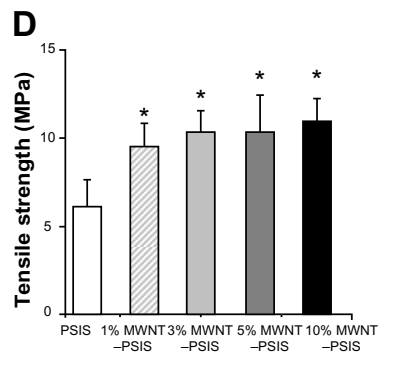

Figure 2 Cumulative release of VEGF 165 in four experimental groups and one control group.

Notes: (A) Sustained release of VEGF ${ }_{165}$ from PSIS scaffolds with varying MWNT content over 7 days in vitro. Determination of HUVEC (B) and fibroblast (C) cell proliferation. (D) In vitro analysis of the tensile strength of scaffolds. *Versus PSIS group, $P<0.05$ in (B-D); " versus I\% MWNT-PSIS group, $P<0.05$ in (B); " versus other experimental groups, $P<0.05$ in $(\mathbf{C})$.

Abbreviations: MWNT, multiwalled carbon nanotubes; PSIS, porcine small intestinal submucosa; HUVEC, human umbilical vein endothelial cell; OD, optical density.

was $143.2 \pm 5.26 \mathrm{pg} / \mathrm{mL}$ and $179.8 \pm 1.37 \mathrm{pg} / \mathrm{mL}$, respectively. This demonstrated that $94.8 \% \pm 3.2 \%$ and $40.7 \% \pm 4.3 \%$ of the loaded amount of $\mathrm{VEGF}_{165}$ had been released from the two groups during the course of the experiment.

The corresponding values in groups $\mathrm{C}$ and $\mathrm{D}$ were $377.44 \pm 6.10 \mathrm{pg} / \mathrm{mL}$ and $460.33 \pm 6.01 \mathrm{pg} / \mathrm{mL}$, respectively, which demonstrated that $50.0 \% \pm 4.1 \%$ and $30.5 \% \pm 3.5 \%$ of the loaded amount of $\mathrm{VEGF}_{165}$ had been released during the experiment. The concentration of $\mathrm{VEGF}_{165}$ released during the first 6 hours in group A was low. Its concentration exceeded that in the control group from the next time point $(P<0.05)$. In other experimental groups, the $\mathrm{VEGF}_{165}$ released was significantly higher than that in the control group over 7 days $(P<0.05)$. The $\operatorname{VEGF}_{165}$ concentration in the composite scaffolds increased significantly when $\mathrm{VEGF}_{165}$-releasing devices were used $(P<0.05)$. Accordingly, group $\mathrm{D}$, which incorporated 10 weight percent $\mathrm{VEGF}_{165}$-releasing devices, released the highest amount of $\mathrm{VEGF}_{165}$ in vitro $(P<0.05)$.

Proliferation of HUVECs in response to the scaffoldderived $\mathrm{VEGF}_{165}$ is shown in Figure 2B. Although group D released the highest concentration of $\mathrm{VEGF}_{165}$, it was found to inhibit HUVEC proliferation $(P<0.05)$. The proliferation of HUVECs in group B and group $C$ increased by 1.6-fold and 2.6-fold, respectively, compared to that of the control group after 7 days of culture $(P<0.05)$. These results indicated that the scaffold containing 5 weight percent $\mathrm{VEGF}_{165}$-releasing devices had optimum ability to stimulate the proliferation of HUVECs in vitro.

\section{Examination of biocompatibility}

Fibroblast proliferation in group A, group B, and group C was not significantly different from that observed in the control group $(P>0.05)$, suggesting that these groups had appropriate scaffolds and good biocompatibility. In contrast, the fibroblast proliferation in group $\mathrm{D}$ was significantly slow and inhibited eventually $(P<0.05$; Figure $2 \mathrm{C})$.

\section{Examination of mechanical properties}

The tensile strength of various composite scaffolds is shown in Figure 2D. The results indicated that the tensile strength of 
the composites did not linearly increase with the increasing of MWNT.

Scaffold containing 5 weight percent of VEGF $_{165}-\mathrm{MWNT}$ exhibited optimal properties in vitro. Therefore, this scaffold was used as the experimental group (VEGF-MWNT-PSIS) for later in vivo studies. The composite scaffold containing the same concentration of MWNT without VEGF ${ }_{165}$ (MWNTPSIS) and the PSIS scaffold served as control groups.

\section{Evaluation of composite scaffold in vivo Postoperative observations}

Rats implanted with scaffolds (Figure 3A-C) showed postoperative recovery from surgery and survived until sacrificed. There were no clinical signs of surgical site infection in

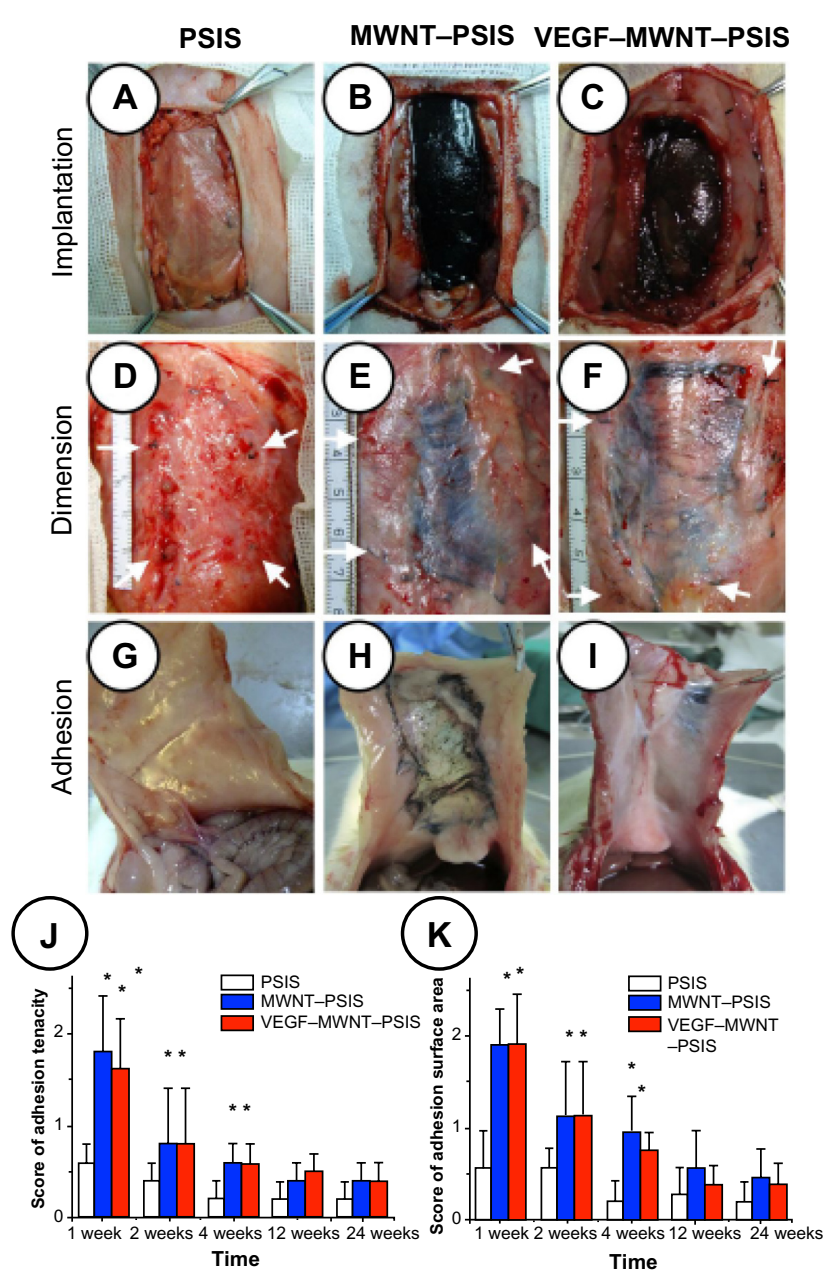

Figure 3 Macroscopic observations of the implanted scaffold.

Notes: Rats were implanted with PSIS, MWNT-PSIS, and VEGF-MWNT-PSIS scaffolds, respectively $(\mathbf{A}-\mathbf{C})$. Rats recovered from surgery after 24 weeks. Sutures are marked by white arrows, indicating contraction or expansion of implants (D-F); meanwhile, adhesion was observed $(\mathbf{G}-\mathbf{I})$ and scored according to adhesion tenacity $(\mathbf{J})$ and surface area $(\mathbf{K})$ analyses of explants, I-24 weeks after implantation. *Versus PSIS explants, $P<0.05$.

Abbreviations: PSIS, porcine small intestinal submucosa; MWNT, multiwalled carbon nanotubes; VEGF, vascular endothelial growth factor. any groups. Examination of explant revealed no herniation around or at the repaired site. Two weeks after surgery, seroma was observed in one rat belonging to the PSIS group and another in the MWNT-PSIS group. Further examination showed evidence of host tissue rupture adjacent to the implanted interface in one animal belonging to the PSIS group 2 weeks after surgery. The rupture led to small intestinal obstructions in parts of the surgical sites.

\section{Macroscopic observations}

White arrows in Figure 3D-F indicate the contraction or expansion of implants surrounded by sutures. In addition, 24 weeks after surgery, the surface area of the PSIS implants $\left(10.22 \pm 0.82 \mathrm{~cm}^{2}\right)$ diminished by $14.8 \%$, while the surface area of MWNT-PSIS $\left(16.32 \pm 1.32 \mathrm{~cm}^{2}\right)$ and VEGF-MWNT-PSIS implants $\left(17.90 \pm 1.56 \mathrm{~cm}^{2}\right)$ increased by $36 \%$ and $49 \%$, respectively $(P<0.05)$. There was no significant difference between the implant surface area of the MWNT-PSIS group and that of the VEGF-MWNTPSIS group $(P>0.05)$.

The composite implants displayed more extensive and dense adhesions between the implants and the peritoneal contents than the PSIS implants within the first 4 weeks. One week after implantation, the tenacity and the surface area of the adhesion scores in the VEGF-MWNT-PSIS group were nearly threefold of that in the PSIS group $(P<0.05)$. However, the adhesions weakened dramatically and dropped to a level comparable to that of PSIS group at 12 weeks postimplantation $(P>0.05)$. At 24 weeks postimplantation, adhesions were negligible. Adhesions were not significantly different between the two composite groups (MWNT-PSIS and VEGF-MWNT-PSIS) throughout the entire experimental periods ( $P>0.05$; Figure $3 \mathrm{G}-\mathrm{K})$.

At 24 weeks postimplantation, all explants were thicker than the original scaffolds $(0.20 \mathrm{~mm} ; P<0.05)$. The thickness of explants in the composites groups (MWNTPSIS $=0.40 \pm 0.03 \mathrm{~mm}$; VEGF-MWNT-PSIS $=0.42 \pm 0.04 \mathrm{~mm}$ ) was higher than that in the PSIS group $(0.28 \pm 0.02 \mathrm{~mm}$; $P<0.05)$. There was no significant difference in the thickness of explants of the two composite groups $(P>0.05)$.

\section{Histological examination of host tissue incorporation}

Host tissue incorporation, defined by new collagen deposition and new vessel growth into the implants, ${ }^{23}$ was determined for both experimental and control groups. Two weeks after implantation, the density of capillary ingrowth was significantly higher in VEGF-MWNT-PSIS $(3.1 \% \pm 1.1 \%)$ than that in PSIS $(1.2 \% \pm 0.3 \%)$ and MWNT-PSIS $(0.7 \% \pm 0.3 \%)(P<0.05)$. 
Neovascularization continued in the VEGF-MWNT-PSIS group until 12 weeks after implantation.

Afterward, similar density of vessels was observed inside the original scaffold implantation sites with no statistical difference among all groups at 24 weeks $(P>0.05$; Figure 4A-J). Masson's trichrome staining revealed that the explants from the VEGF-MWNT-PSIS group induced more collagen deposition from 4-24 weeks than the other two groups $(P<0.05)$, while there was no significant difference in collagen deposition between the PSIS and the
MWNT-PSIS groups. All the explants showed similar collagen organization after implantation ( $P>0.05$; Figure 5).

\section{Examination of mechanical properties after implantation}

All scaffolds in this study failed at the interface between the scaffold and the abdominal wall. Therefore, the reported values do not represent the strength of the actual material, but the integration process at the level of the interface with the surrounding tissue - termed the "strength of incorporation". ${ }^{24,25}$
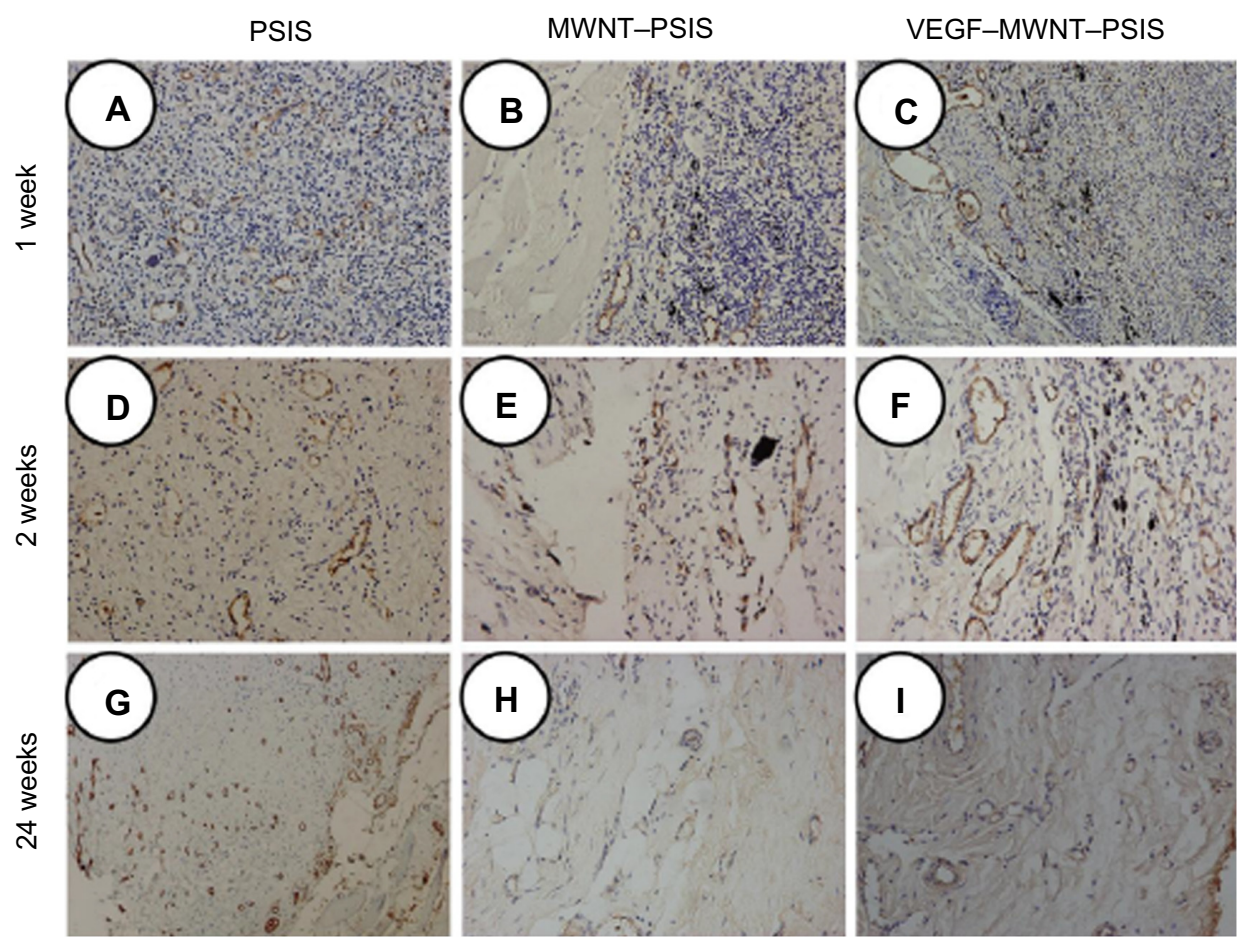

J

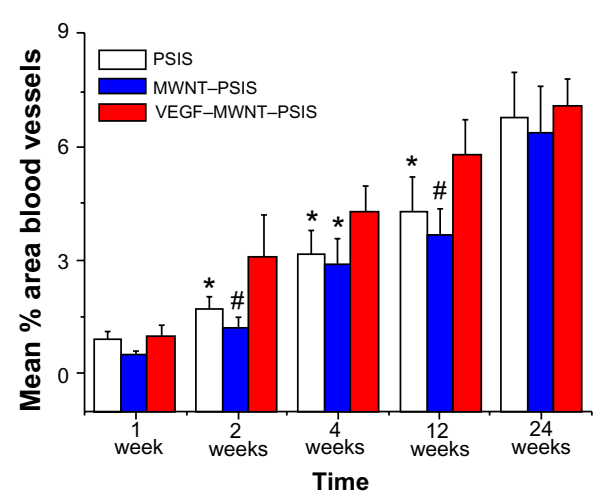

K

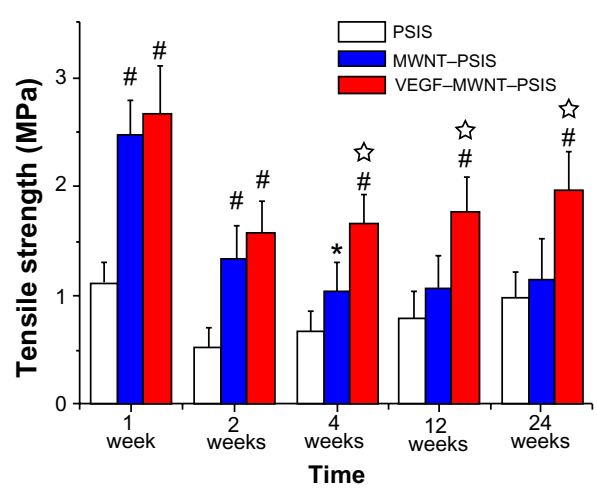

Figure 4 Microscopic analysis of explants stained for von Willebrand factor and tensile strength evaluation.

Notes: Newly formed blood vessels were sparse at the center of non-VEGF-containing scaffolds; whereas, active neovascularization occurred at the center of the VEGFMWNT-PSIS scaffold during the first 2 weeks after implantation $(\mathbf{A}-\mathbf{F}, \times 200)$. A similar amount of blood vessels was observed at the site of original scaffold after 24 weeks $(\mathbf{G}-\mathbf{I}, \times 200)$. Density of blood vessels was estimated by histological semiquantitative analysis of the explants (J); tensile strength in vivo was recorded (K) starting I-24 weeks after implantation. ${ }^{* P}<0.05$ versus VEGF-MWNT-PSIS explants in $(\mathbf{J}) ;{ }^{*} \boldsymbol{P}<0.0$ I versus VEGF-MWNT-PSIS explants in $(\mathbf{J}) ;{ }^{*}<0.05$ versus PSIS explants in (K); ${ }^{*}<<0.0$ I versus PSIS explants in $(\mathbf{K})$; 2 次 $<0.05$ versus MWNT-PSIS explants in (K).

Abbreviations: PSIS, porcine small intestinal submucosa; MWNT, multiwalled carbon nanotubes; VEGF, vascular endothelial growth factor. 


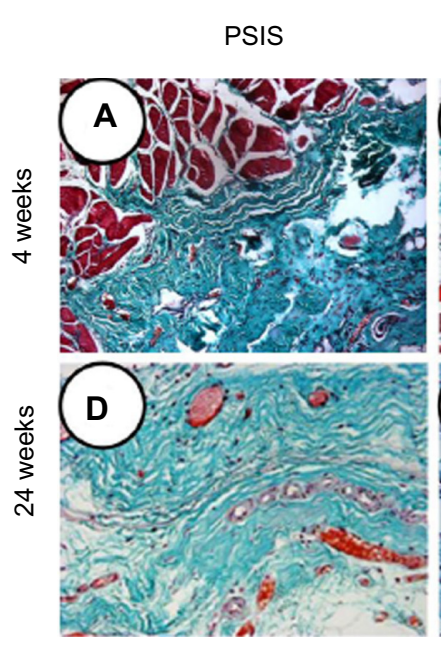

G

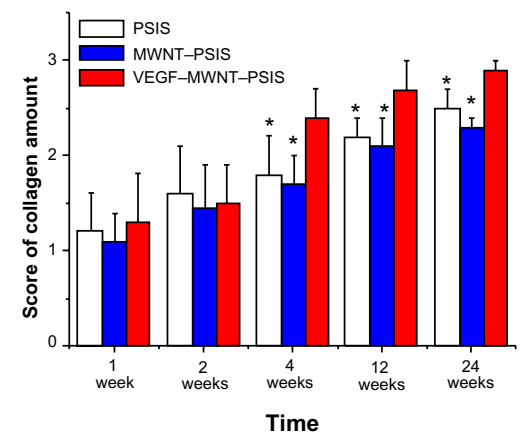

MWNT-PSIS

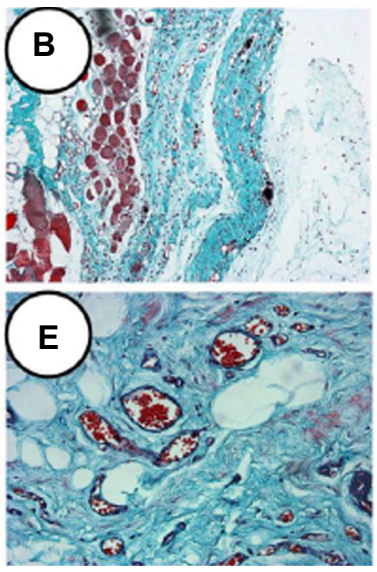

VEGF-MWNT-PSIS

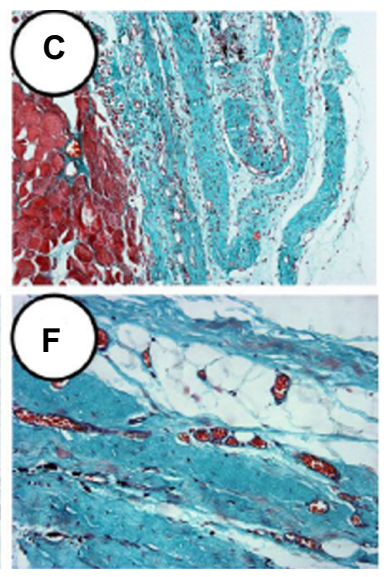

H

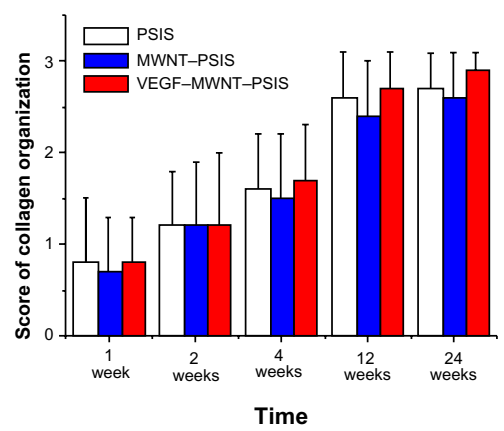

Figure 5 Microscopic analysis of explants with Masson's staining and tensile strength examination.

Notes: An organized pocket of collagen deposition at the interface of PSIS and MWNT-PSIS explants was observed 4 weeks postimplantation, while distinct lamellar sheets of organized collagen were observed in the VEGF-MWNT-PSIS explants during the same period $(\mathbf{A}-\mathbf{C} \times 100)$. All the implants were replaced by oriented bundles of collagenous tissue at the center of the repair sites 24 weeks after implantation $(\mathbf{D}-\mathbf{F}, \times 200)$. Collagen organization $(\mathbf{G})$ and collagen amount $(\mathbf{H})$ were evaluated by histological semiquantitative analysis of explants starting from I week up to 24 weeks after implantation versus $* P<0.05$, VEGF-MWNT-PSIS explants in (G).

Abbreviations: MWNT, multiwalled carbon nanotubes; VEGF, vascular endothelial growth factor; PSIS, porcine small intestinal submucosa.

The strength of incorporation of all explants after implantation was significantly lower than that of initial scaffolds throughout the evaluation periods. These values decreased to a minimum (below that of the natural abdominal wall) after 2 weeks, and it gradually increased thereafter to a maximum at 24 weeks postimplantation. MWNT-PSIS and VEGF-MWNT-PSIS explants maintained considerably higher tensile strength within the initial 2 weeks compared to that of the PSIS explants $(P<0.01)$. However, the difference between them was statistically insignificant $(P>0.05)$. At later time points, the VEGF-MWNT-PSIS explants exhibited greater strength of incorporation than the other two groups $(P<0.05)$. The MWNTPSIS explants eventually exhibited a strength of incorporation comparable to that of PSIS explants ( $P>0.05$; Figure 4K).

\section{Inflammatory response}

Microscopic examination revealed the presence of mixed infiltrating inflammatory cells after implantation (Figure 6).
As evident from the scores of foreign body cells and polymorphonuclear cells at the interface, the MWNT-PSIS and the VEGF-MWNT-PSIS explants showed pronounced inflammatory responses, which were significantly greater than that in PSIS explants 1 week after implantation $(P<0.05)$. The inflammatory responses were evident throughout the period of evaluation, with peak level observed at 2 weeks postimplantation in composite explants. Inflammatory responses dropped to negligible levels by the end of the study $(P<0.05)$. The inflammatory response was not significantly different among the two composites explants $(P>0.05)$.

\section{Discussion}

When used in repairing abdominal wall defects, PSIS serves as a scaffold, providing mechanical support to initially counteract intra-abdominal pressure and promoting host tissue incorporation to compensate for the loss of mechanical strength with the degradation of implants during 


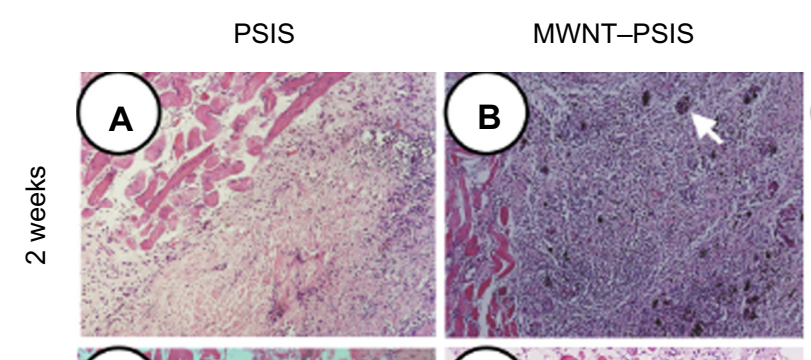

VEGF-MWNT-PSIS
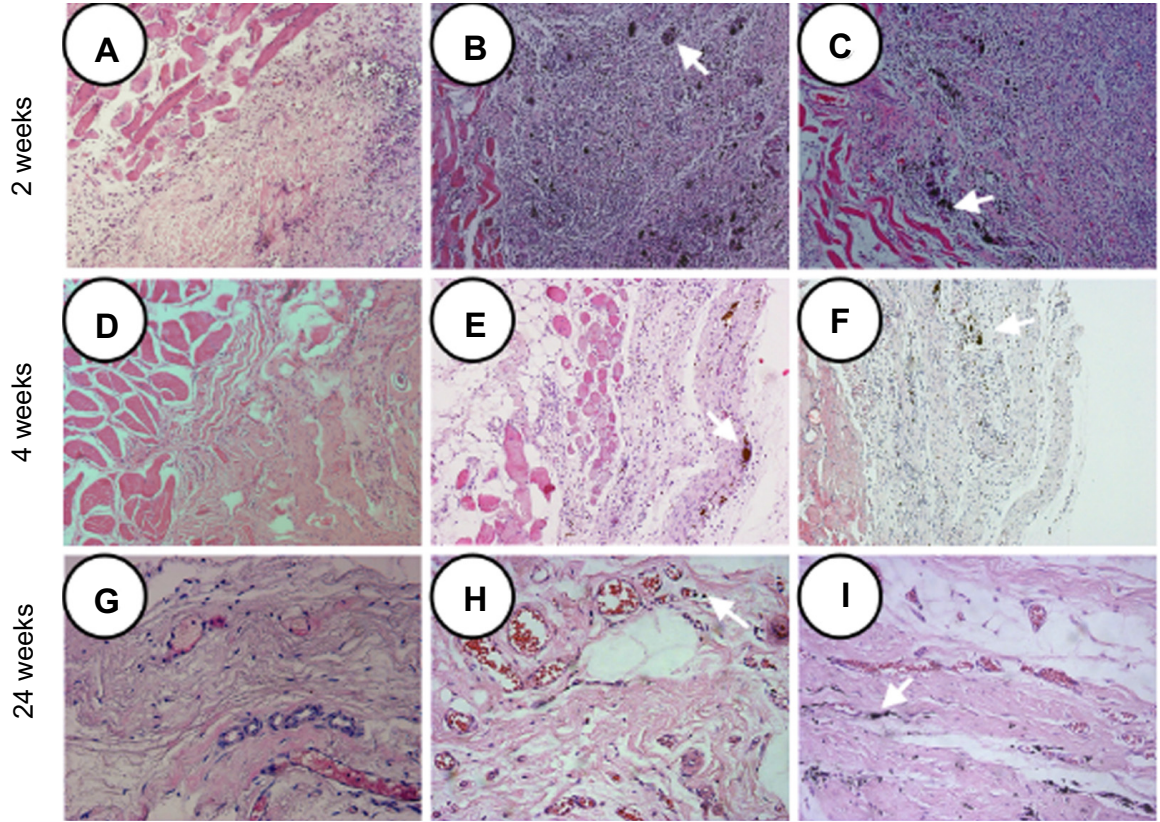

J

K

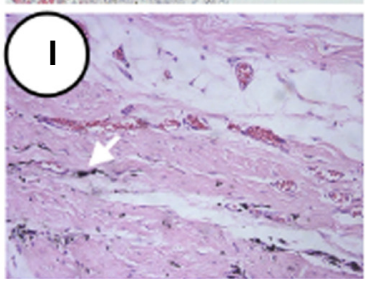

$\mathbf{L}$
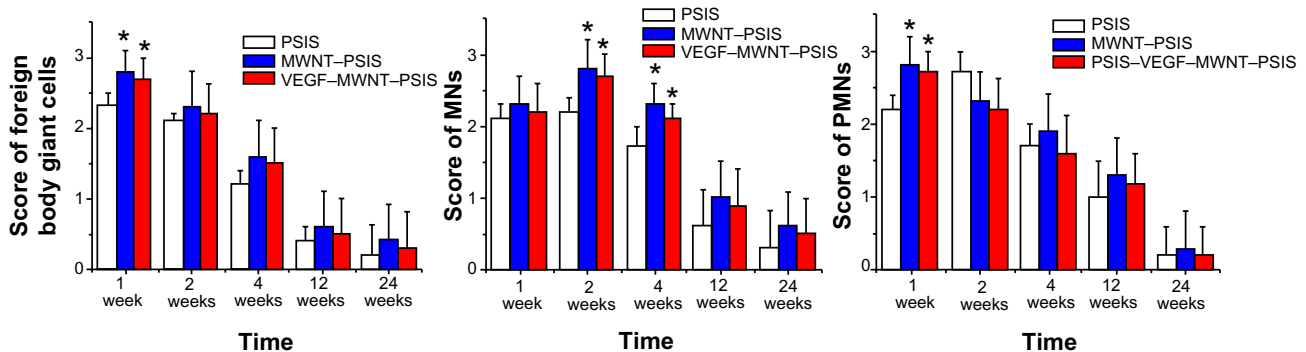

Figure 6 Microscopic analysis of the explants following hematoxylin and eosin staining.

Notes: Infiltration of mixed inflammatory cells was observed at the interface between PSIS implant and the surrounding tissues at 2 weeks, while pronounced inflammatory cells infiltration was observed surrounding the perimeter of composite implants, and CNT residuals were evident as the black spots $(\mathbf{A}-\mathbf{C} \times 100)$. Sparse inflammatory cells were observed in PSIS implants, while chronic inflammatory process was observed in composite explants after 4 weeks, especially in MWNT-PSIS (D-F). Blood vessels and host tissue incorporation with fibroblast and endothelial cell invasion was observed; only a few scaffolds remained at the center of the constructs. Residues were evident in composite scaffolds at week 24 (G-I; $\times 200)$. Histological semiquantitative analysis for foreign body giant cells $(\mathbf{J})$, polymorphonuclear $(\mathbf{K})$ leukocytes, and mononuclear cells (L) in explants I-24 weeks after implantation. $* P<0.05$ versus PSIS explants in (J-L) and versus VEGF-MWNT-PSIS explants in (E and $\mathbf{F}$ ).

Abbreviations: MWNT, multiwalled carbon nanotubes; VEGF, vascular endothelial growth factor; PSIS, porcine small intestinal submucosa; MNs, mononuclear cells; PMNs, polymorphonuclear cells.

subsequent periods. However, the insufficient early vascularization in PSIS scaffolds following implantation leads to limited host tissue incorporation and may cause herniation and hernia recurrence in clinical study. The scaffold developed in this study supported sustained release of VEGF ${ }_{165}$, accelerated angiogenesis and neovascularization, and promoted effective host tissue incorporation.

Our study proved that due to their three-dimensional hollow core structure and PLGA-coating, functionalized MWNT can be used as carriers of $\mathrm{VEGF}_{165}$ to ensure its sustained release. VEGF $_{165}$ was loaded in the tube and on the wall of MWNT, though the percentage distributed in each compartment was not clear. The HRTEM graphs confirmed that the MWNT were coated with PLGA. The plasma functionalization technique used in our study was similar to that described by Shi et al, ${ }^{17}$ who prepared PLGA-coated carbon nanotubes (CNT) as binders to carry paclitaxel. To our knowledge, this is the first time that plasma surface functionalization was used to construct a $\mathrm{VEGF}_{165}$-releasing device that was then used to repair abdominal wall defects. Our results demonstrated that the $\mathrm{VEGF}_{165}$ concentration in composite scaffolds was significantly higher than that in PSIS scaffolds over 7 days. The result indicates the scaffolds in group D obtained the highest cumulative released concentration and lowest released rate in all groups over 7 days. The reversed appearance in group D implies that the 
high concentration of VEGF ${ }_{165}-$ MWNT impregnated retards the effective released manner. Apparently, the speculation can be supported by the tendency that - with the increase of VEGF-MWNT in the scaffold - the released rate of VEGF-MWNT decreased, while the cumulative released concentration of that increased in our study. With the concentration of separating VEGF-MWNT increased in scaffold, it caused the local aggregation of MWNT, ${ }^{26}$ and more VEGF-MWNT device was incorporated into the inner of the three-dimensional scaffold and released more slowly with scaffold degradation.

Our study showed that the $\mathrm{VEGF}_{165}$ released from VEGF-MWNT-PSIS led to a higher blood vessel density in the explant and an early-stage boost of neovascularization. However, the real-time concentrations of $\mathrm{VEGF}_{165}$ released in vivo from the implants were not determined. To the best of our knowledge, many imaging strategies capable of monitoring VEGF levels quantitatively have been reported. ${ }^{27,28}$ However, the absence of sophisticated methods in situ to monitor and distinguish the concentration of the unreleased VEGF from the released VEGF respectively may hinder the mechanistic investigations in the sustained release of the composite scaffold. Hence, more experimental data of labeled $\mathrm{VEGF}_{165}$ and MWNT released with degradation in vivo is required to provide a means for further understanding the released mechanism of the composite scaffold. In the present study, when compared with controls, a higher blood vessel density was observed in the VEGF-MWNTPSIS explants during the 12 weeks of postimplantation. Compared to the PSIS and the MWNT-PSIS implants, early neovascularization of the VEGF-MWNT-PSIS implant stimulated more collagen production. This was further supported by the observation of well-vascularized and organized fibrous connective tissues in the VEGFMWNT-PSIS explants.

The key factor for host tissue incorporation is early and stable neovascularization, which is critical for the delivery of oxygen and nutrients to actively regenerating tissues, and restores endothelial cell signaling. ${ }^{29}$ Further, the tensile strength of new fascia was reinforced from the regenerated tissue in response to collagen deposition.

Our study showed that early and thorough host tissue incorporation achieved using VEGF-MWNT-PSIS in vivo was the main source of tensile strength at the interface with the host tissues in the abdominal wall defects repair. Within the first 2 weeks of postoperation, implants containing MWNT exhibited greater strength of incorporation when compared to the PSIS implants, owing to the structure of the nanocomposite support. However, the tensile strength of all three groups reached a nadir 2 weeks following implantation when the implant had undergone only limited incorporation. Thus, the degradation rate of the implant outpaced the rate of collagen deposition and the replacement of fibrous connective tissue. The strength of incorporation of all the tested explants at this time point was below the values of natural abdominal wall in rats $(2.26 \pm 0.43 \mathrm{MPa})$, although no hernia was observed.

However, the VEGF-MWNT-PSIS implant facilitated new vessel ingrowth and collagen deposition that reinforced the tensile strength starting from 4 weeks to the end of the study. The strength of incorporation of PSIS and MWNTPSIS explants increased slightly, owing to the limited and persistent host tissue incorporation.

Although we successfully introduced the MWNT as carriers for the sustained release of $\mathrm{VEGF}_{165}$, some adverse effects were observed. First, the MWNT-containing implants caused pronounced inflammatory responses within the first 4 weeks after implantation. In contrast, PSIS implants only induced a mild inflammatory response. We believe that the enhanced inflammatory response induced more severe adhesion compared to PSIS implants. However, the inflammatory responses subsided with degradation, host tissue incorporation, and replacement of the implants at the repair site in all three groups. Moreover, PSIS is a natural and completely absorbable biomaterial, while MWNT are nonabsorbable and underwent rapid, first-order clearance from the blood compartment via renal excretion, although the CNT has been proved to have little influence on cell proliferation within safe concentration by various study. ${ }^{30-32}$ The interaction between MWNT and VEGF in our system is noncovalent, which is considered a favorable factor for efficient drug release. Our system is not perfect, but we deem it a meaningful attempt to improve the performance of natural biological material, and a more ideal carrier will be adapted to the sustained release system in the near future.

\section{Conclusion}

The preparation and use of functionalized MWNT-carrying $\mathrm{VEGF}_{165}$ led to accelerated tissue repair by ensuring sustained growth factor release. To our knowledge, this is the first time this novel VEGF ${ }_{165}$ delivery device was integrated with PSIS to construct a composite scaffold that improved neovascularization - thereby achieving enhanced host tissue remodeling and repair. Our results pave the way for the development of advanced materials for drug delivery and tissue regeneration. 


\section{Acknowledgments}

The authors thank Daxiang Cui for technical support. This work was supported by the Medicine and Engineering Crossed Foundations, Shanghai Jiao Tong University (grant number YG2010MS50 and YG2013MS61).

\section{Disclosure}

The authors report no conflicts of interest in this work.

\section{References}

1. Eriksen JR, Gögenur I, Rosenberg J. Choice of mesh for laparoscopic ventral hernia repair. Hernia. 2007;11(6):481-492.

2. Leber GE, Garb JL, Alexander AI, Reed WP. Long-term complications associated with prosthetic repair of incisional hernias. Arch Surg. 1998;133(4):378-382.

3. Iannitti DA, Hope WW, Norton HJ, et al. Technique and outcomes of abdominal incisional hernia repair using a synthetic composite mesh: a report of 455 cases. J Am Coll Surg. 2008;206(1):83-88.

4. Mahmoud Uslu HY, Erkek AB, Cakmak A, et al. Incisional hernia treatment with polypropylene graft: results of 10 years. Hernia. 2006;10(5): 380-384.

5. Vaught JD, Kropp BP, Sawyer BD, et al. Detrusor regeneration in the rat using porcine small intestinal submucosal grafts: functional innervation and receptor expression. J Urol. 1996;155(1):374-378.

6. Badylak S, Kokini K, Tullius B, Whitson B. Strength over time of a resorbable bioscaffold for body wall repair in a dog model. J Surg Res. 2001;99(2):282-287.

7. Ko R, Kazacos EA, Snyder S, Ernst DM, Lantz GC. Tensile strength comparison of small intestinal submucosa body wall repair. J Surg Res. 2006;135(1):9-17.

8. Poulose BK, Scholz S, Moore DE, et al. Physiologic properties of small intestine submucosa. J Surg Res. 2005;123(2):262-267.

9. Petter-Puchner AH, Fortelny RH, Walder N, et al. Small intestine submucosa (SIS) implants in experimental IPOM repair. J Surg Res. 2010;161(2):264-271.

10. Lai JY, Chang PY, Lin JN. Body wall repair using small intestinal submucosa seeded with cells. J Pediatr Surg. 2003;38(12):1752-1755.

11. Chen Y, Liu L. Modern methods for delivery of drugs across the bloodbrain barrier. Adv Drug Deliv Rev. 2012;64(7):640-665.

12. Shireman PK, Hampton B, Burgess WH, Greisler HP. Modulation of vascular cell growth kinetics by local cytokine delivery from fibrin glue suspensions. J Vasc Surg. 1999;29(5):852-861; discussion 862.

13. Ehrbar M, Djonov VG, Schnell C, et al. Cell-demanded liberation of VEGF121 from fibrin implants induces local and controlled blood vessel growth. Circ Res. 2004;94(8):1124-1132.

14. Wilcke I, Lohmeyer JA, Liu S, et al. VEGF(165) and bFGF proteinbased therapy in a slow release system to improve angiogenesis in a bioartificial dermal substitute in vitro and in vivo. Langenbecks Arch Surg. 2007;392(3):305-314.
15. Zisch AH, Lutolf MP, Hubbell JA. Biopolymeric delivery matrices for angiogenic growth factors. Cardiovasc Pathol. 2003;12(6):295-310.

16. Besteman K, Lee JO, Wiertz FGM, Heering HA, Dekker C. Enzymecoated carbon nanotubes as single-molecule biosensors. Nano Lett. 2003;3(6):727-730.

17. Shi D, Guo Y, Dong Z, et al. Quantum-Dot-Activated Luminescent Carbon Nanotubes via a Nano Scale Surface Functionalization for in vivo Imaging. Adv Mater. 2007;19(22):4033-4037.

18. Liu J, Rinzler AG, Dai H, et al. Fullerene pipes. Science. 1998;280(5367): 1253-1256.

19. Guo Y, Shi D, Cho H, et al. In vivo Imaging and Drug Storage by Quantum Dot Conjugated Carbon Nanotubes. Advanced Functional Materials. 2008;18(17):2489-2497.

20. Liu Z, Tang R, Zhou Z, Song Z, Wang H, Gu Y. Comparison of two porcine-derived materials for repairing abdominal wall defects in rats. PLoS One. 2011;6(5):e20520.

21. Jenkins SD, Klamer TW, Parteka JJ, Condon RE. A comparison of prosthetic materials used to repair abdominal wall defects. Surgery. 1983;94(2):392-398

22. Badylak S, Kokini K, Tullius B, Simmons-Byrd A, Morff R. Morphologic study of small intestinal submucosa as a body wall repair device. J Surg Res. 2002;103(2):190-202.

23. MacLeod TM, Sarathchandra P, Williams G, Sanders R, Green CJ. Evaluation of a porcine origin acellular dermal matrix and small intestinal submucosa as dermal replacements in preventing secondary skin graft contraction. Burns. 2004;30(5):431-437.

24. Meyer T, Schwarz K, Ulrichs K, Höcht B. A new biocompatible material (Lyoplant) for the therapy of congenital abdominal wall defects: first experimental results in rats. Pediatr Surg Int. 2006;22(4):369-374.

25. Gaertner WB, Bonsack ME, Delaney JP. Experimental evaluation of four biologic prostheses for ventral hernia repair. J Gastrointest Surg. 2007;11(10):1275-1285.

26. Smart SK, Cassady AI, Lu GQ, Martin DJ. The biocompatibility of carbon nanotubes. Carbon. 2006;44(6):1034-1047.

27. Kang CM, Koo HJ, Lee KC, et al. A vascular endothelial growth factor 121 (VEGF(121))-based dual PET/optical probe for in vivo imaging of VEGF receptor expression. Biomaterials. 2013;34(28):6839-6845.

28. Chang SK, Rizvi I, Solban N, Hasan T. In vivo optical molecular imaging of vascular endothelial growth factor for monitoring cancer treatment. Clin Cancer Res. 2008;14(13):4146-4153.

29. Mondalek FG, Ashley RA, Roth CC, et al. Enhanced angiogenesis of modified porcine small intestinal submucosa with hyaluronic acid-poly (lactide-co-glycolide) nanoparticles: from fabrication to preclinical validation. J Biomed Mater Res A. 2010;94(3):712-719.

30. Singh R, Pantarotto D, Lacerda L, et al. Tissue biodistribution and blood clearance rates of intravenously administered carbon nanotube radiotracers. Proc Natl Acad Sci U S A. 2006;103(9):3357-3362.

31. Tian F, Cui D, Schwarz H, Estrada GG, Kobayashi H. Cytotoxicity of single-wall carbon nanotubes on human fibroblasts. Toxicol in Vitro. 2006;20(7):1202-1212.

32. Meng J, Song L, Meng J, et al. Using single-walled carbon nanotubes nonwoven films as scaffolds to enhance long-term cell proliferation in vitro. J Biomed Mater Res A. 2006;79(2):298-306.
International Journal of Nanomedicine

\section{Publish your work in this journal}

The International Journal of Nanomedicine is an international, peerreviewed journal focusing on the application of nanotechnology in diagnostics, therapeutics, and drug delivery systems throughout the biomedical field. This journal is indexed on PubMed Central, MedLine, CAS, SciSearch ${ }^{\circledR}$, Current Contents ${ }^{\circledR} /$ Clinical Medicine,

\section{Dovepress}

Journal Citation Reports/Science Edition, EMBase, Scopus and the Elsevier Bibliographic databases. The manuscript management system is completely online and includes a very quick and fair peer-review system, which is all easy to use. Visit http://www.dovepress.com/ testimonials.php to read real quotes from published authors. 